\title{
Muß Mathematik angewandt werden?
}

\author{
von Ernst Kleinert
}

Das Thema „Mathematik und Anwendung " enthält Fragen verschiedenen Gewichts; als erste die, wie wir verstehen sollen, daß Mathematik überhaupt angewandt werden kann. Immer wieder ist doch staunenswert, wie sie mit ihren Deduktionen so lange neben der Welt herlaufen und dann doch wieder mit ihren Resultaten in sie hineinspringen kann [1]. Untergeordnet, aber mitunter drängender ist die Frage, ob Mathematik nach Anwendung streben sollte, oder schärfer, sich durch Anwendung zu legitimieren habe. Natürlich ist als Nächstes zu fragen, was wir als Anwendung, vollends als legitimierende Anwendung gelten lassen wollen. Wir erinnern uns an das Scherzwort, die Zahlentheorie sei eine nützliche Wissenschaft, weil man nämlich mit ihr promovieren könne. Der Leser wird auch selbst wissen, was dem Kategorientheoretiker zu antworten ist, welcher behauptet, seine Disziplin sei die am meisten angewandte. Aber zählt ein Schachprogramm als Anwendung der mathematischen Logik?

1

Nun tritt uns eine Institution entgegen, die allbekannte Dichotomie und Konkurrenz „reine“ versus „angewandte" Mathematik. Schon sprachlich ist die Einteilung mißglückt; suggeriert sie doch, daß die Mathematik, die angewandt wird, etwas anderes sei als ganz normale Mathematik, der man im übrigen nichts hinzufügt, wenn man sie „rein“ nennt.

Unhaltbar aber auch die übliche Zuordnung der Disziplinen, denn man kann sich auf recht abgehobene Art mit Wahrscheinlichkeitstheorie und auf sehr praxisnahe mit Primzahlen beschäftigen, so daß zwischen die „wahrhaft reine“ und die „wirklich angewandte" Mathematik wenigstens noch eine „reine angewandte" und eine ",angewandte reine" einzuschieben wären. Der Unterschied zwischen Theorie und Praxis geht quer durch die Fakultäten und ist eher in den Vorlieben der Einzelnen begründet als in der Sache.

Kein Stück Mathematik wird allein dadurch wirklich welthaltig, daß es sich als „angewandt " klassifizieren läßt. Auch mathematische Modellbildung, nur unter schwacher Beweispflicht stehend, kann l'art pour l'art werden (und natürlich gibt es eine mathematische Theorie der mathematischen Modellbildung). Immer besteht die Gefahr, daß die Annahmen, die den Kalkül handhabbar machen, das Modell unrealistisch werden lassen.

Solches veranlaßte die Verfasser eines Lehrbuchs der mathematischen Biologie [2] zu der sarkastischen Bemerkung, es handle sich mitunter eher um Anwendungen der Biologie in der Mathematik als umgekehrt. Und wie sollen wir die immer wieder genannten Anwendungen schätzen, welche Primzahlen und elliptische Kurven in der Krytographie gefunden haben? Es ist keineswegs ausgemacht, ob es, aufs Gan- ze gesehen, ein Vorteil ist, wenn Geldinstitute oder Nachrichtendienste voreinander Geheimnisse haben.

2

Von beiden Seiten wachsen nun Brücken. Die umfassende Bearbeitung eines arithmetischen Schemas erfordert die Theorie der partiellen Differentialgleichungen und Greenschen Funktionen ebenso wie die der Gruppen, Ringe und Körper; ein System ganzzahliger Polynome definiert ein arithmetisches Objekt durch seine ganzen oder rationalen, ein analytisches durch seine reellen oder komplexen Lösungen. Daß letzte Wahrheit nur da liegen kann, wo alle Wege zusammenkommen, ist keine neue Einsicht; aber erst in unseren Tagen werden die begrifflichen Mittel bereitgestellt, die ein Abzielen darauf möglich machen, und seitdem müssen sich auch Zahlentheoretiker für Pseudodifferentialoperatoren interessieren.

Von der andern Seite greifen die Spekulationen der Physiker seit einiger Zeit in mathematische Bereiche über, deren Anwendungsfähigkeit man noch vor wenigen Jahrzehnten für Utopie erklärt hätte [3]. Sie initiieren oder befördern auch neue, wie die Theorie der Quantengruppen. Wie weit die Vermischung fortgeschritten ist, zeigt vielleicht am schönsten die Lösung des „moonshine“-Rätsels. Die Theorie der unendlichdimensionalen Liealgebren, aus der reinen Mathematik hervorgegangen, wurde erst von den Physikern aufgegriffen und erwies sich dann als der Schlüssel zum Verständnis jener mysteriösen Verquickung von Zahlen, die MacKay beobachtet hatte, der Koeffizienten der Modulfunktion und der Darstellungsgrade der größten sporadischen Gruppe; ein Phänomen, welches sicherlich in der höchsten Zinne des Elfenbeinturms anzusiedeln war. So taucht die Einheit aller mathematischen Betrachtung, vordem mehr eine 
Floskel oder ein Versprechen, am Horizont der Spekulation als Wirklichkeit auf.

3

Daß Mathematik danach trachten solle, mit der Welt in Verbindung zu bleiben, ist allerdings auch entschieden zurückgewiesen worden; am bekanntesten wohl in Hardys „Apology“. Es fällt freilich schwer, in dieser Schrift mehr zu sehen als ein mehr oder minder witziges Glaubens- und Geschmacksbekenntnis. Wenn er die angewandte Mathematik kurzerhand für „dull“ erklärt, so genügt, dem zu begegnen, die trockene Versicherung des Gegenteils. Die provozierende Erklärung, in der die Schrift gipfelt, nämlich, nie etwas Nützliches getan zu haben, erfreut uns immerhin als passende Antwort auf die Verdikte eines zu eng genommenen Utilitarismus.

Was aber hier entscheidet, ist die verborgene Inkonsistenz einer solchen Position. Kann ein ernsthafter Mensch sein Leben einer Tätigkeit widmen, von der er überzeugt ist, sie sei in einem absoluten Sinne nutzlos, erfülle sich ohne Rest in sich selbst? Selbst wenn er verschmäht oder für unmöglich hält, seine Mathematik in die Welt zu bringen, wird er nicht dennoch im Geheimen glauben, daß sie in irgendeinem höheren Sinne mit der Welt verbunden sei, ja gar, wenn er platonisch gestimmt ist, daß sie der „eigentlichen " Welt angehöre? Und wenn er sich für einen reinen Ästheten erklärt, wird er nicht dennoch glauben, daß die Harmonie der Begriffe, die er vermehrt, etwas an sich Wichtiges sei, dessen Legitimation nicht allein darin liegen kann, daß ein Orden von Tüftlern nicht müde wird, sich damit abzumühen?

Daß auch Hardy so empfand, zeigen seine Bemühungen, die Mathematik gegen das Schachspiel abzusetzen. Allerdings bedürfen seine Kriterien der $s e$ riousness, substance and depth einer besseren Begründung.

Wer aber sagt: ich tue etwas, das getan werden muß, das der Menschheit, oder auch nur mir selbst aufgegeben ist, unterwirft sich damit einem umfassenderen Anspruch; und wenn er über dessen Art, Herkunft und Ziel keine Rechenschaft ablegen kann, so ist das noch kein Grund, an einem telos zu verzweifeln. Er akzeptiert, daß sein Tun in einem Dienst steht; das muß ihn nicht hindern, zu widersprechen, wenn andere ihm die Dienstbarkeit definieren wollen. „Zur höheren Ehre des Menschlichen Geistes " — das war Jacobis Formel, die sich Weil zu eigen machte [4]. Darin liegt eine Verpflichtung; man würde eben nicht gelten lassen, wenn einer behauptete, er spiele Schach um dieser Ehre willen.

Am Ende kann ein Nachdenkender sich nicht damit zufriedengeben, daß der Geist, der ihm alles bedeutet, nur in sich selbst Bestand haben soll; er will ihn in die Welt eingehen sehen, vielleicht in eine andere als diese, eine künftige. Vielleicht steht hinter diesem Zwang, immer höher zu steigen, immer mehr zu erfahren von den Notwendigkeiten des eigenen Geistes, auch der geheime Wunsch, mit einem anderen Geist in Berührung zu treten.

"Zahlen sind grundlegendes Denkgut der Menschheit; über sie möglichst viel herauszufinden, ist Pflicht." Ja, wenn man noch hoffen könnte, irgendwann dieses Suchen und Finden abschließen zu können. Seit Gödel aber ist diese Hoffnung abgeschnitten, und Pflicht ist vielmehr, zu fragen, welche Informationen über Zahlen relevant sind. Besonders deutlich, geradezu schmerzhaft zeigt das negative Resultat zu Hilberts 10. Problem, daß auch sehr konkrete und natürlich scheinende Probleme den naiven Zugriff zurückweisen können. Trotzdem tut man manchmal, als sei ein endlicher Fortschritt, ein neuer gelöster Fall eine Annäherung an das Ganze, das doch unendlich fern bleibt.

Nicht auszuweichen ist also der Frage nach leitenden Gesichtspunkten, die aus dem unausschöpfbaren Möglichen das Wichtige sondern; das, was wir wissen müssen, das, was uns weiterbringt. Die Kantische Forderung, Wissenschaft im Bereich möglicher Erfahrung zu halten, wird uns hier von der mathematischen Grundlagenforschung selbst in unerwarteter Weise aufgezwungen. Treten wir einen Schritt zurück und versuchen wir, die Rolle der Mathematik im Ganzen der conditio humana in den Blick zu bekommen.

Die mathematischen Grundbegriffe über Zahl, Raum, Gesamtheiten und Beziehungen zwischen solchen sind kategorialer Natur; sie gehören zu den Mitteln, vermöge welcher wir Weltphänomene auffassen und gedanklich bearbeiten. Die Axiome der Mathematik formulieren einige der einfachsten Gesetzmäßigkeiten, unter denen diese Kategorien stehen, und aus denen sich andere ableiten lassen: die Abfolge der Zahlen mit ihren Beziehungen der Größe und Teilbarkeit, die Dimension, Metrik und Orientierung des Raumes, der Schematismus von Teil und Ganzem und des Enthaltenseins der Teile, die Logik der Funktionen mit ihren verschiedenen Aspekten von Zuordnung, Abbildung oder Transport. In diesem Sinne enthält die Mathematik den beweisbaren Teil der Philosophie. Da alle Erfahrung unter Kategorien steht, muß auch jede in der Erfahrung sich zeigende Gesetzlichkeit in der Sprache jener Grundbegriffe formulierbar sein, ja sogar aus ihnen deduzierbar, also der Erfahrung vorwegzunehmen, a priori geltend. 
Wo nun die mathematische Betrachtung Axiome und erste Begriffe bildet, hebt sie nicht fertige Strukturen aus dem Erfahrenen, sondern bearbeitet dieses in charakteristischer Weise. Der mathematische Zahlbegriff unterscheidet sich in wenigstens dreierlei Hinsicht von allen wirklichen Anzahlen: er postuliert ein Zahlsystem, in dem jede einzelne Zahl ihren Platz hat; dieses ist homogen, seine Mitglieder sind nur hinsichtlich ihrer Bezüge untereinander, aber nicht als Individuen an sich unterscheidbar, die einzelnen Zahlen sind scharf voneinander getrennt, gehen nicht ineinander über. Das Erste ist Generalisierung, das Zweite Abstraktion, das Dritte Idealisierung. Diese mathematische Präparation öffnet die kategorialen Gesetzlichkeiten für Fragestellungen, die aus dem ursprünglich Gegebenen hinausführen; sie schafft ein freies Feld für gedankliche Operationen, die am Konkreten schnell eine Grenze fänden. Und sogleich macht sich der wehende Geist seine eigenen Probleme, die ihn nicht mehr loslassen, fasziniert sich selbst in der uns allen bekannten Weise.

\section{5}

Bleiben wir, um das zu illustrieren, bei den Zahlen. Sie sind fundamental für unsere Auffassung und Bearbeitung der Welt; das Multiplizieren ist fundamental für den Umgang mit Zahlen; die Primzahlen sind fundamental für die multiplikative Struktur. Aber sonderbar: Transitivität findet nicht statt; in der Welt ausgezeichnet sind kleine Zahlen und ihre Vielfachen, die Primeigenschaft tritt kaum hervor; welche Rolle spielt es, daß die Finger einer Hand, die Tage einer Woche von primer Anzahl sind?

Hier können wir etwas deutlicher sehen. Für die Multiplikation ist die Primzerlegung eine Substruktur, die man nicht zu kennen braucht, so wie Jourdain Prosa redete, ohne es zu wissen; und beim Dividieren muß man ohnehin mit Brüchen und Resten leben; die Anwendung des euklidischen Algorithmus gibt keinen Aufschluß über die Primzerlegung. So wird, was ein Zahlentheoretiker für das „wahre Wesen“ der Zahlen halten könnte, von der Welt schlichtweg ignoriert.

Hier liegt ein Rätsel. Die Elemente der Mathematik sind dicht an der Welt; die mathematische Betrachtung springt jäh von ihr ab, obwohl sie uns natürlich, ja zwingend erscheint und wir unabweisbar fühlen, es müsse damit „etwas auf sich haben“. Sollen wir denken müssen, diese Organisation unserer Vernunft leite uns in ewig unfruchtbare Spekulationen?

6

Von den Vorgaben der conditio humana, unserer kategorialen Verfassung, können wir nicht abstrahieren; sie gehen in jede Artikulation bereits ein. Der Weg, den wir gehen können, führt über den Ausbau des kategorialen Gefüges, die Entwicklung der in ihm angelegten Gesetzmäßigkeiten; von ihm ist die Mathematik ein Teil, dessen deduktive Autarkie uns lage getäuscht hat. Gäbe es eine Formalisierung mathematischer Bedeutsamkeit, so gäbe es auch darüber einen Satz vom Gödeltyp; gibt es aber keine, so bedeutet dies eben, daß uns die Mathematik selbst nicht sagen kann, was wir sie fragen sollen, daß also der Ursprung unserer Fragen anderswo liegen muß. Offenbar ist notwendig, den mathematischen Teil der Vernunft zu entfalten; er arbeitet durch Isolation von Begriffsgefügen; aber in der Isolierung belassen, fällt er der Verzweiflung anheim, beweist sich selbst seine Ohnmacht. Er muß am Ende eingehen in eine mathesis universalis, in das Denken und Trachten der Menschen im Ganzen.

Daß sie vor diesem Hintergrund handle, ist von der Mathematik zu fordern; daß sie Sorge trage, in diesem Ganzen bestehen zu können. Das scheint mir alles, was sich mit Verbindlichkeit sagen läßt, und ist vage genug; oft wird solche Sorge nicht mehr leisten können, als die Suche oder auch nur Hoffnung nach dem Brückschlag zur Welt nicht aufzugeben. Unsinnig wäre die Forderung, daß jede Vermehrung des Wissens sich sogleich in eine Vermehrung des Handelnkönnens umzusetzen habe; unsinnig aber auch der Anspruch, daß jede Faszination durch Phänomene der Mathematik, jede Ahnung vermeintlichen Tiefsinns bereits eine potentiell unendliche Bearbeitung legitimiere.

Faktisch übt die mathematical community eine Art Selbstkontrolle, indem sie Standardfragen in den Mittelpunkt stellt und jeden Beitrag befragt, was er zu diesen beiträgt. Die Standardfragen wiederum sind diejenigen, die aus dem Gesichtspunkt der vorgängigen Forschung als die natürlicherweise zu fragenden erscheinen. Der Fortgang ist aber nicht zwingend und darin liegt einerseits die Möglichkeit des Fehlgehens und Scheiterns, andererseits das Feld einer Verantwortlichkeit, die darauf achtet, daß Mathematik nicht zum Glasperlenspiel verkommt.

Damit freilich der mathematische Genius sein Bestes leiste, muß man ihm genügend Auslauf lassen, ihm am langen Bande das Flattern gönnen. Man gestatte ihm ruhig, um ihn bei guter Laune zu halten, Hardys Ansichten. Den Errichtern der Elfenbeintürme ist die Menschheit zu Dank verpflichtet; aber sie wird sich nicht abhalten lassen, diese in Baulichkeiten aus mehr irdischem Material einzubeziehen.

Schließlich weht der Geist, von wannen er will; auch untergeordnete Fragen können große Einsichten hervorbringen; Dieudonné sah darin gar „das Paradies der Mathematiker" [5]. Gauß lehnte es ab, sich mit Fermats Problem zu beschäftigen [6]; doch in den 
Händen Kummers wurde es eine Eingangspforte zur algebraischen Zahlentheorie.

$$
7
$$

Klassisches Vehikel der Anwendung ist das mathematische Modell, dessen wirkungsweise Hertz mit bekannten Worten beschrieben hat: „Wir machen uns innere Scheinbilder oder Symbole von den äußeren Gegenständen, und zwar machen wir sie von solcher Art, daß die denknotwendigen Folgen der Bilder stets wieder die Bilder der naturnotwendigen Folgen der abgebildeten Gegenstände sind " [7]. Der „reinen" Mathematik wird nun vorgehalten, daß sie nicht Bilder von Dingen oder Vorgängen macht oder wenigstens mittelbar auf solche abzielt. Eine leichte Antwort liegt bereit: vielleicht sind ja die Dinge und Vorgänge, auf die jene Mathematik abzielen könnte, noch nicht in Erscheinung getreten. Die Beispiele der Relativitätstheorie und Quantenmechanik zeigen, daß der Vorgriff der Mathematik ins Reich reiner Strukturen gute Aussichten hat, nachträglich „Bestätigung und Recht und Licht" zu erhalten. Vielleicht muß ja der Vorgriff soweit ins Leere hinausreichen, wenn sehr große Aufgaben anstehen; wie ein Hammerschlag umso stärker trifft, je weiter ausgeholt wurde. Man mache sich nur klar, von welch letzter Verstiegenheit die theoretischen Grundlagen der Kernspaltung zu sein scheinen; und man erinnere sich, daß es metamathematische, ins Philosophische greifende Probleme waren, die eine Klärung der Begriffe der Entscheidbarkeit und Berechenbarkeit erzwangen und so dem maschinellen Rechnen den Grund legten.

Um das Besondere zu fördern, muß sich Wissenschaft von ihm lösen und ins Allgemeine gehen. Kraft der Axiomatisierung weiß sie das Besondere nicht mehr, aus dem sie stammt; aber eben darin liegt die Möglichkeit, sich unerwartet aufs Neue zu besondern. Für die Physik, die ihre mathematischen Methoden längst nicht mehr in der Anschauung verankern kann, ist die Mathematik eine Art Struktur-Baukasten geworden; das wäre nicht möglich, wenn sie immer nur auf schon gestellte Fragen geantwortet hätte.

Diese Erklärung lädt freilich zum Mißbrauch ein, zu überzogener Hypotheken-Aufnahme auf künftige Anwendung. Es gibt eine notwendige, in der Sache begründete Weltferne und eine parasitäre, dem Unvermögen oder der Willkür entstammende. Da man zu den großen Problemen nichts beitragen kann, leitet man aus ihnen solche ab, die man lösen kann. Ist ein Kreis von Fragen einmal anerkannt, so auch ein Umkreis; die Hilfsmittel-Funktion iteriert sich, es entsteht eine Hierarchie von Legitimität. Jeder Begriff ist ein Derivat ursprünglicher Begriffe, aber vielleicht ein sehr verdünntes; und es stellt sich die Fràge nach dem noch tolerablen Grade der Verdünnung. Manchmal erscheint sie in der Tat homöopathisch. Jene notwendige Weltferne aber ist eine scheinbare, denn sie rückt, auf die Länge, unseren Begriff von der Welt zurecht, erzwingt ein neues Paradigma. Wir mußten, um ihrer Herr zu werden, weiter ausholen als erwartet.

Das gilt im Hinblick auf Anwendung wie für die mathematische Theorie selbst. Um die mathematischen Weltteile der Arithmetik und der Geometrie zu einer Sphäre zu vereinigen, bedurfte es der gewaltigen Abstraktionen, mit denen Grothendieck seine Zeit in Erstaunen versetzte. Aber was heute noch manchem als eine Wolkenlandschaft erscheinen mag, könnte einmal ebenso selbstverständlich das Ausgangsfeld mathematischen Denkens und Anschauens sein, wie es das einfachere Reich der Zahlen und Figuren so lange gewesen ist.

\section{8}

Von anderer Art als jene klassischen sind die neueren Anwendungen algebraischer und arithmetischer Strukturen in Kombination und Kommunikation, beim Suchen, Ordnen, Planen, Regeln und Verschlüsseln. Es ist hier, wo scheinbar „reinste“ Gebilde der Mathematik, Primzahlen, Kurven über endlichen Körpern, arithmetische Gruppen [8] ihre kombinatorischen Aspekte enthalten und in Anwendungen eintreten. Hier sind die charakteristischen Probleme von Hause aus Mathematik und müssen nicht eigens modelliert werden. Es gab sie auch immer schon; sie waren nur nicht virulent, solange der Fluß von Daten und Gütern, der Verkehr im weitesten Sinne noch ad hoc und in Handarbeit bewältigt werden konnte. Das Ende dieses Urzustands brachte zugleich, mit explodierender Theorie und Praxis elektronischen Kalkulierens, das Werkzeug zur Lösung der vergrößerten Probleme.

Das klassische Modell enthält ein Bild der Welt und macht Prognosen über die Welt. Hier jedoch entfaltet sich der Teil menschlichen Handelns, der von sich aus mathematisch ist und nur des Organs bedurfte. Endlich entbunden, schießt er in alle Bereiche ein; die reißende Schnelligkeit dieses Siegeszugs macht sichtbar, wie weit das mathematische Handeln hinter der mathematischen Betrachtung zurückgeblieben war. Erst damit wird der ungeheure Umbau der Lebenswelt möglich, den wir jetzt erfahren und der uns von früheren Befindlichkeiten unwiderruflich abschneidet. Die Mathematik dringt gleichsam selbst in die Welt vor, indem die mathematischen Möglichkeiten des Planens und Handelns zu den allgegenwärtigen werden und nur noch dulden, was sich ihnen anpaßt. Der Schlüssel macht sich alles zum Schloß. 
Damit beginnt auch die Frage nach „rein “ oder „angewandt" ihre Konturen zu verlieren. Wenn jemand sporadische Gruppen benützte, um ein Computerspiel zu basteln, so würde man das schwerlich für eine die Gruppenforschung legitimierende Anwendung halten. Was aber, wenn alles darauf flöge und Millionengewinne hereinkämen? Dann wäre es den Gruppen ergangen wie den großen Primzahlen, die man für eine public-key-Verschlüsselung benötigt und für ein paar tausend Dollar kaufen kann. Wer vermöchte vorauszusagen, was für Strukturen in den heraufkommenden virtuellen Welten figurieren werden?

Die Menschheit definiert sich ihre Zwecke selbst, und deren Entfaltung bringt immer wieder mit sich, daß Mittel zu Zwecken werden; man sehe nur auf das Beförderungs- und Kommunikationswesen. Warum soll nicht einmal ein pythagoreisches Zeitalter einsetzen, das über uns die Nase rümpft, weil wir nicht genug für die Lehre von den Schiefkörpern getan haben? Wenn die Numerologie wieder zu Ansehen kommt (und man bedenke, was für Möglichkeiten sie heute hat), werden die Arithmetiker zu Oberpriestern.

\section{9}

Wir sind ausgegangen von der Frage, ob die Mathematik an der Welt bleiben soll, und angelangt bei der Feststellung, daß die Welt sich anschickt, mathematisch zu werden. Soll der Geist dem Leben dienen, oder das Leben dem Geist? Wir können darin übereinkommen, daß der Geist lebendig werden, das Leben aber sich vergeistigen solle. Das kann man als die beiden Seiten eines Prozesses auffassen, den Teilhard de Chardin als Bildung der Noosphäre beschrieben hat: die Verdichtung der Gedankenströme um den Erdball bis hin zur Konstitution eines Gesamtbewußtseins [9]. Hier erscheint es als Symbol hohen Ranges, daß die Mathematik immer kleinere Teile von Materie mit Strukturen ausstattet und dazu bringt, für uns zu rechnen und uns zu antworten.

Die legitime Frage nach Anwendung entspringt einem Denken aufs Ganze. Geläufiger ist ihre Umkehrung, die Frage nach der Theorie zu bekannten Phänomenen. Verstehen wollen, was wir sehen und erfahren, ist von nie bestrittener Legitimität, auch da, wo weitere Verwendungen solchen Verstehens nicht in Sicht sind. Eher wird uns die Homologietheorie praktischen Nutzen bringen als die Kosmologie. Um den Kreis zu schließen, sollten wir wünschen, daß, wo es etwas Großes zu verstehen gibt, dieses auch in die Wirklichkeit einzugehen fähig sei. Ein Ereignis erster Größenordnung bietet hier eine unzerstörbare Quelle der Zuversicht: daß ein Bedürfnis der Geometrie, die Berechnung von Tangenten und Inhalten, dieselbe Infinitesimalrechnung hervorbrachte wie das
Bedürfnis der Mechanik, Bewegungsvorgänge mathematisch zu beschreiben. Eine mathematische Theorie, die sowenig selbstverständlich ist, daß sie eine Inkubationszeit von fast zweitausend Jahren benötigte, erwies sich als der gemeinsame Kern einer „reinen“ Theorie wie einer äußerst handhaften Praxis.

Mit der ersten mathematischen Betrachtung trat der Geist aus dem Stand der Unschuld, der Kreatur, die "mit allen Augen das Offene" sieht [10]. Der Weg zurück ist versperrt. Aber solange jene Verantwortlichkeit, von der oben die Rede war, nicht nachläßt, dürfen wir hoffen, daß die Rationalität, in der Mathematik sich entfaltend und darstellend, schließlich, im Durchgang durch unendliche Erkenntnis, den Weg findet zu einer Stelle, an der, wie Kleist spekulierte [11], die Enden der ringförmigen Welt und das Paradies wieder offen ist.

\section{Verweise:}

[1] Das bekannteste Staunen stammt von E. Wigner: „The Unreasonable Effectiveness of Mathematics in the Natural Sciences", Comm. Pure and Appl. Math. vol. XIII (1960), 1-14. Um die Pointe zu verschärfen, entwickelt Wigner allerdings eine sonderbare Auffassung vom Gange mathematischer Begriffsbildung: „Most more advanced mathematical concepts ... were so devised that they are apt subjects on which the mathematician can demonstrate his ingenuity and sense of formal beauty" (S. 3). Das ist wohl kaum eine adäquate Beschreibung der Motive, die zur Entwicklung der Infinitesimalrechnung, der Galoisschen Theorie, der Riemannschen Geometrie usw. geführt haben.

[2] J. Hofbauer und K. Sigmund, Evolutionstheorie und dynamische Systeme, Berlin und Hamburg 1984 (Vorwort).

[3] Es sei verwiesen auf den Band „From Number Theory to Physics", M. Waldschmidt et al. (Hrsg.), Springer 1992.

[4] A. Weil, The Future of Mathematics, Am. Math. Monthly 57 (1950), 295-306.

[5] J. Dieudonné, M. Loi, R. Thom (Hrsg.), Penser les mathématiques, Editions du Seuil (1982), S. 27.

[6] Siehe seinen Brief vom März 1816 and die Franz. Akad. d. Wissensch.

[7] H. Herz, Die Prinzipien der Mechanik, Leipzig 1894, S. 1.

[8] Für die letzteren sehe man nach in A. Lubotzky, Discrete Groups, Expanding Graphs and Invariant Measures, Birkhäuser 1994 .

[9] Teilhard de Chardin, L'Avenir de l'Homme, Editions du Seuil 1959, S. 199ff.

[10] R. M. Rilke, Achte Duineser Elegie.

[11] H. v. Kleist, Über das Marionettentheater.

\section{Adresse des Autors:}

Ernst Kleinert

Mathematisches Seminar

Universität Hamburg

20146 Hamburg

ekleinert@math.uni-hamburg.de 